THE ASTROPHYSICAL JournaL, 338:618-629, 1989 March 15

(C) 1989. The American Astronomical Society. All rights reserved. Printed in U.S.A.

\title{
QUASAR-GALAXY ASSOCIATIONS WITH DISCORDANT REDSHIFTS AS A TOPOLOGICAL EFFECT. II. A CLOSED HYPERBOLIC MODEL
}

\author{
HELIO V. FAGUNDES \\ Instituto de Física Teórica, Universidade Estadual Paulista, São Paulo, Brazil \\ Received 1988 April 28; accepted 1988 August 23
}

\begin{abstract}
This is the second of two papers developing a conjecture that might solve the quasar redshift controversy. The quasar-galaxy or quasar-quasar associations with discordant redshifts reported by Arp and others would be explained as the effect of multiple image production in a cosmological model with nontrivial space topology. The model here explored has the same local metric as Friedmann's open model, but the latter's infinite space is replaced by a closed space, constructed as usual from a polyhedron with faces pairwise ideritified. The results exhibit, semiquantitatively, some of the characteristics of the mentioned associations, and are suggestive of other effects such as an inhomogeneous distribution of QSO images on the sky. But no actual fitting of data is attempted, for these are too rich and varied, and probably will require a more complex topology for their explanation than the one used here. So the present model will be seen as a prototype for future developments in this direction. A possible connection with the ideas of small universe of Gott and Ellis is hinted at.

Subject headings: cosmology - galaxies: clustering - galaxies: redshifts - quasars
\end{abstract}

\section{INTRODUCTION}

The first part of this research was published a few years ago (Fagundes 1985). It presented the view that the quasar redshift controversy could be resolved if the infinite space of Friedmann's open cosmological model were replaced by a closed hyperbolic manifold (CHM). That paper, henceforth referred to as QGAI, treated the problem in the unrealistic but simplified context of a two-dimensional region of space, or threedimensional region of spacetime. Although the unfamiliar mathematical machinery there introduced caused some concern, I hope it has paved the way for the class of more realistic models which will be exemplified in this paper. Here I shall tone down the mathematics, at least in the main text; some subtle points, as well as most calculations, are left to the Appendices.

The model developed in this paper has the spacetime metric of a Friedmann-Robertson-Walker (FRW) model of negative spatial curvature, but the comoving three-dimensional space is represented by a $\mathrm{CHM}$ rather than by Lobachevskian, or hyperbolic, space $\left(\mathrm{H}^{3}\right)$. The CHM is an identification space, obtained by identifying, or "gluing," pairs of faces of a fundamental polyhedron in $\mathrm{H}^{3}$, here a hyperbolic icosahedron (see $\S \mathrm{II})$. As $\mathrm{H}^{2}$ in QGAI, here $\mathrm{H}^{3}$ remains as the space of potential images of the sources in the CHM. The observed approximate isotropy and the postulated global homogeneity are reinterpreted as an isotropy and homogeneity of the space of images $\mathbf{H}^{3}$. In the language of geometers, the latter is tessellated, or honeycombed, into replicas of the fundamental polyhedron (FP). The sources are located in this fundamental cell; points in the replicas, up to the observational horizon, are potential images of those sources-potential because there might be no source at the corresponding real point, or a source there might not have been radiating at the appropriate time.

As discussed theoretically in QGAI, \$§ III, IV, and observationally by Sokolov and Shvartsman (1974), present data do not contradict the idea that space has a nontrivial topology like that of the model developed below. For a general discussion on the topology of cosmic space see Ellis (1971).
The associations of quasars and galaxies with discordant redshifts reported by Arp and others (see Burbidge 1981 for a review) are reinterpreted as near-conjunctions of multiple images of single sources, whose rays would have traveled different paths from the source, but arrived at Earth from about the same direction on the sky. The redshifts are thus cosmological, as in the standard FRW models, but the distance parameters are those of the rays' paths; the smallest of these may be said to be the source's distance from us.

Repeated images also result from simpler FRW models, such as the three-toroidal one in Fang and Sato (1983), Demianski and Lapucha (1987), Fagundes and Wichoski (1987), and others. But the data on associations of quasars and galaxies with discordant redshifts, which are at the core of the controversy, seem more readily simulated by closed (that is, compact and boundless; see Seifert and Threlfall 1980) hyperbolic models, which also predict an inhomogeneous distribution of groups of QSO images; see $\S \mathrm{V}$ below.

As stated in QGAI ( $\S \mathrm{I}, \mathrm{VI})$, I expected to find quickly a CHM that would fit the observations. But it turned out that this is a project of wider scope than anticipated, because of the large number of possibilities. So we have to adopt the emerging methodology of computer simulations (see, for example, Hut and Sussman 1987), making a systematic, as automated as possible, search among the models of this class. The model here detailed is thus to be viewed as a prototype for that larger project.

The density ratio in this prototype is again taken to be $\Omega=0.1$, as in QGAI and Fagundes (1986). This value may look like a conservatively low estimate, but it is still a real possibility for the average distribution of matter and radiation, if the former is predominantly "ordinary," rather than "exotic," matter. In any case, this value is maintained because, combined with the chosen CHM, it has allowed a semiquantitative simulation of some observations.

The next section describes the theoretical framework, and $\S$ III, IV show the processes by which multiple images, particularly conjunctions, are produced. 
II. A FRIEDMANN MODEL WITH CLOSED, HYPERBOLIC SPATIAL SECTIONS

In a notation similar to that of Landau and Lifshitz (1975), I will write Friedmann's hyperbolic metric (an FRW metric with cosmological constant $\Lambda=0$ and density ratio $\Omega<1$ ) as

$$
d s^{2}=a^{2}(\eta)\left(d \eta^{2}-d \sigma^{2}\right),
$$

where

$$
\begin{gathered}
a(\eta)=a_{0}(\cosh \eta-1), \\
d \sigma^{2}=d \chi^{2}+\sinh ^{2} \chi\left(d \theta^{2}+\sin ^{2} \theta d \varphi^{2}\right),
\end{gathered}
$$

and the parameter $\eta$ is related to time by

$$
c t=a_{0}(\sinh \eta-\eta) .
$$

The cosmological redshift $Z(I)$ of an image $I$ at distance $\chi=\chi_{I}$ is given by

$$
1+Z(I)=\frac{a\left(\eta_{0}\right)}{a\left(\eta_{0}-\chi_{I}\right)},
$$

where $\eta_{0}=\eta\left(t_{\text {now }}\right)$ is the radius of the observational horizon in the comoving metric.

Now, while the global structure of spacetime in Friedmann's hyperbolic model may be represented by $\mathrm{H}^{3} \times \mathrm{T}_{+}$, where $\mathrm{H}^{3}$ is hyperbolic space and $T_{+}$is the positive time semiaxis, in our case it is $\mathrm{CHM} \times \mathrm{T}_{+}$, where CHM is a closed hyperbolic manifold or space. The chosen CHM is one of the three given by Best (1971) having a regular hyperbolic isosahedron as fundamental polyhedron (FP). It was already used by the author in a concise report on the possibility of multiple images of our own Galaxy (Fagundes 1986). This FP is drawn in Figure 1, and its geometry is explained in Appendix A. Table 1 gives the numbering of its faces. The faces to be identified or "glued" are those whose numbers differ by 10 . Also, the order of the vertices in the faces is maintained in the identification. For example, ICA $\rightarrow$ ABC implies $\mathrm{I} \rightarrow \mathrm{A}, \mathrm{C} \rightarrow \mathrm{B}, \mathrm{A} \rightarrow \mathrm{C}$.

TABLE 1

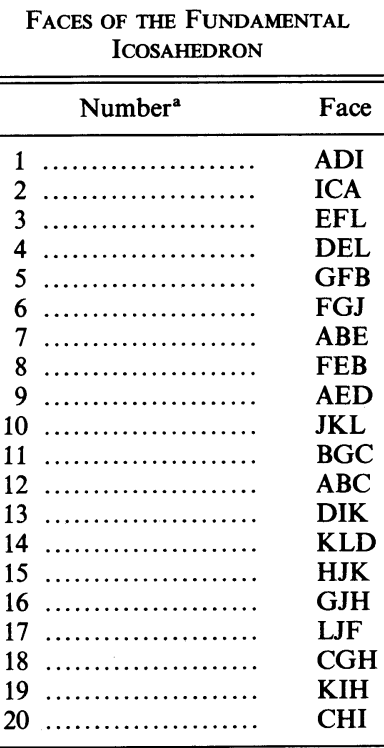

a The numbering indicates the identification of faces: face $\mathrm{N}$ is glued to face $\mathrm{N}+10$.

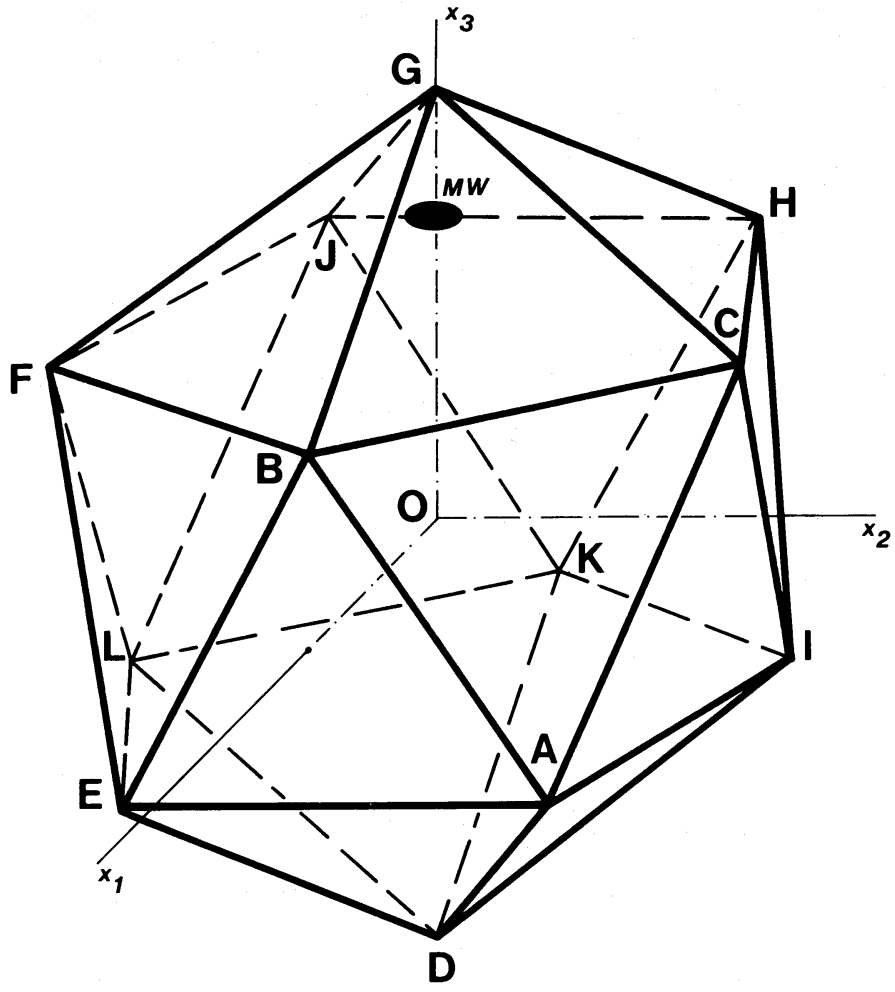

Fig. 1.-The fundamental polyhedron of the prototype model is a hyperbolic regular icosahedron, here represented by a Euclidean icosahedron in cavalier projection. "MW" is the Milky Way Galaxy, located at $(0,0$, tanh 0.75) in this map. Dashed circle is the section plane $x_{2} \mathrm{O} x_{3}$ of the unit ball onto whose interior Bolyai-Lobachevsky's space $\mathrm{H}^{3}$ is compressed.

As in QGAI, it is convenient to use a compact representation of hyperbolic space. Here I shall use Klein's model, which maps $\mathrm{H}^{3}$ onto the interior of a ball of unit radius in ordinary space, and assigns Cartesian coordinates $x=\left(x_{i}\right), i=1-3$, to its points, with the correspondence

$$
\begin{aligned}
& x_{1}=\tanh \chi \sin \theta \cos \varphi, \\
& x_{2}=\tanh \chi \sin \theta \sin \varphi, \\
& x_{3}=\tanh \chi \cos \theta,
\end{aligned}
$$

to coordinates $(\chi, \theta, \varphi)$. Its great advantage is that Lobatchevskian straight lines and planes are mapped by their Euclidean counterparts. The distance between two points $x, y$ is (see Coxeter 1965, p. 209)

$$
d(x, y)=\cosh ^{-1}\left[\frac{1-x \cdot y}{\left(1-x^{2}\right)^{1 / 2}\left(1-y^{2}\right)^{1 / 2}}\right] .
$$

If one of the points is the coordinate center $\mathrm{O}$, say $\boldsymbol{y}=\mathbf{0}$, then it is easier to invert equations $(2.6 \mathrm{a})-(2.6 \mathrm{c})$ to get

$$
d(\boldsymbol{x}, \mathbf{0})=\chi=\tanh ^{-1}|\boldsymbol{x}| .
$$

Figures 1-4 are all centered at the geometric center $O$ of the FP. The position of Earth is denoted by MW (for Milky Way Galaxy) on Figure 1. It was chosen at distance $\chi_{E}=0.75$ in the positive $x_{3}$ direction; hence, its Klein coordinates are $(0,0$, tanh 0.75 ). Note that a CHM is locally homogeneous, so that equations $(2.1)-(2.8)$ are valid for coordinates centered on any observer's position, here MW. But it is not globally homoge- 
neous, a fact that required a reinterpretation of the homogeneity postulate, as explained in QGAI. See also $\S \mathrm{V}$ below.

We also need Earth-centered coordinates $x_{i}^{\prime}$. They are derived in Appendix $\mathbf{A}$ and made explicit by the formulae (where $\beta=\tanh 0.75, \gamma=\cosh 0.75$ )

$$
\begin{aligned}
& x_{1}^{\prime}=\frac{x_{1}}{\gamma\left(1-\beta x_{3}\right)}, \\
& x_{2}^{\prime}=\frac{x_{2}}{\gamma\left(1-\beta x_{3}\right)}, \\
& x_{3}^{\prime}=\frac{x_{3}-\beta}{1-\beta x_{3}},
\end{aligned}
$$

which, curiously enough, look like those of a relativistic velocity transformation (see Fagundes 1986). The distance $\chi^{\prime}$ from Earth is given by equation (2.8) with $\boldsymbol{x}^{\prime}$ substituted for $\boldsymbol{x}$, and I shall use a theoretical longitude and latitude:

$$
\begin{aligned}
& \text { lon }=\varphi^{\prime}=\tan ^{-1}\left(x_{2}^{\prime} / x_{1}^{\prime}\right), \\
& \text { lat }=90^{\circ}-\theta^{\prime}=90^{\circ}-\cos ^{-1}\left(x_{3}^{\prime} /\left|\boldsymbol{x}^{\prime}\right|\right),
\end{aligned}
$$

with the ranges $\theta^{\circ} \leq$ lon $<360^{\circ},-90^{\circ} \leq$ lat $\leq 90^{\circ}$.

\section{THE HONEYCOMBING OF SPACE AND THE MULTIPLE IMAGES} OF A GIVEN SOURCE

The formation of a CHM by face identification implies, by Poincarés theorem, the tessellation, or honeycombing of $\mathrm{H}^{3}$ into replicas of the fundamental polyhedron (see QGAI, § III). A generator in this process is a rigid motion of $\mathrm{H}^{3}$ that takes the FP to a neighbor replica, and the number of the contact face is used to specify the motion (see Table 1 ). There are 20 generators, and generator $G_{k}$ acts on the FP to produce the neighbor cell $G_{k}(\mathrm{FP})$, which contacts the original FP through face $k$ in the latter. For example, $G_{13}(\mathrm{FP})$ and FP have face 13, or DIK in Figures $1-4$, in common.

In Appendix A a matrix formalism is developed that represents points in hyperbolic space by associating them with points in Minkowski space, and then the generators are expressed by Lorentz transformations familiar from special relativity. The 20 generator matrices are listed at the end of that Appendix.

Figure 2 shows the process of multiple image generation. There the Galaxy (MW) is treated as a source, and its position is displaced downward for clarity. The idea, already developed in Fagundes (1986) and Fagundes and Wichoski (1987), is that our Galaxy might have been a powerful source at high $Z$. In Figure $2 \mathrm{MW}$ sends a ray toward a point $M$ on face 6 ; this ray comes back to us from $M^{\prime}$ on face 16 , producing an image $\mathrm{MW}_{16}$. To find the parameters $\left(Z\right.$, lon, lat) for $M W_{16}$, we first find its Minkowski coordinates by motion $G_{16}$; the coordinates of $\mathrm{MW}$ are $(0,0, \sinh 0.75$, cosh 0.75$)$, and writing this as a column matrix $X$, the motion is performed by the matrix equation $X_{16}=G_{16} X$, from which we get $O$-centered Klein coordinates $x_{16, i}=X_{16, i} / X_{16,4}$ (see Appendix A). The Earthcentered coordinates $\boldsymbol{x}^{\prime}$ are given by equations (2.9a)-(2.9c). The length of the looping ray $\mathbf{M W}\left(\mathbf{M}=\mathbf{M}^{\prime}\right) \mathbf{M W}$ in comoving space is the same of that of the apparent ray $\mathrm{MW}_{16}-\mathrm{MW}$, which is $\chi^{\prime}=\tanh ^{-1}\left|x^{\prime}\right|=1.0676$, and from equation (2.5) we get $Z\left(\mathrm{MW}_{16}\right)=2.23$. Finally equations $(2.10 \mathrm{a})-(2.10 \mathrm{~b})$ give

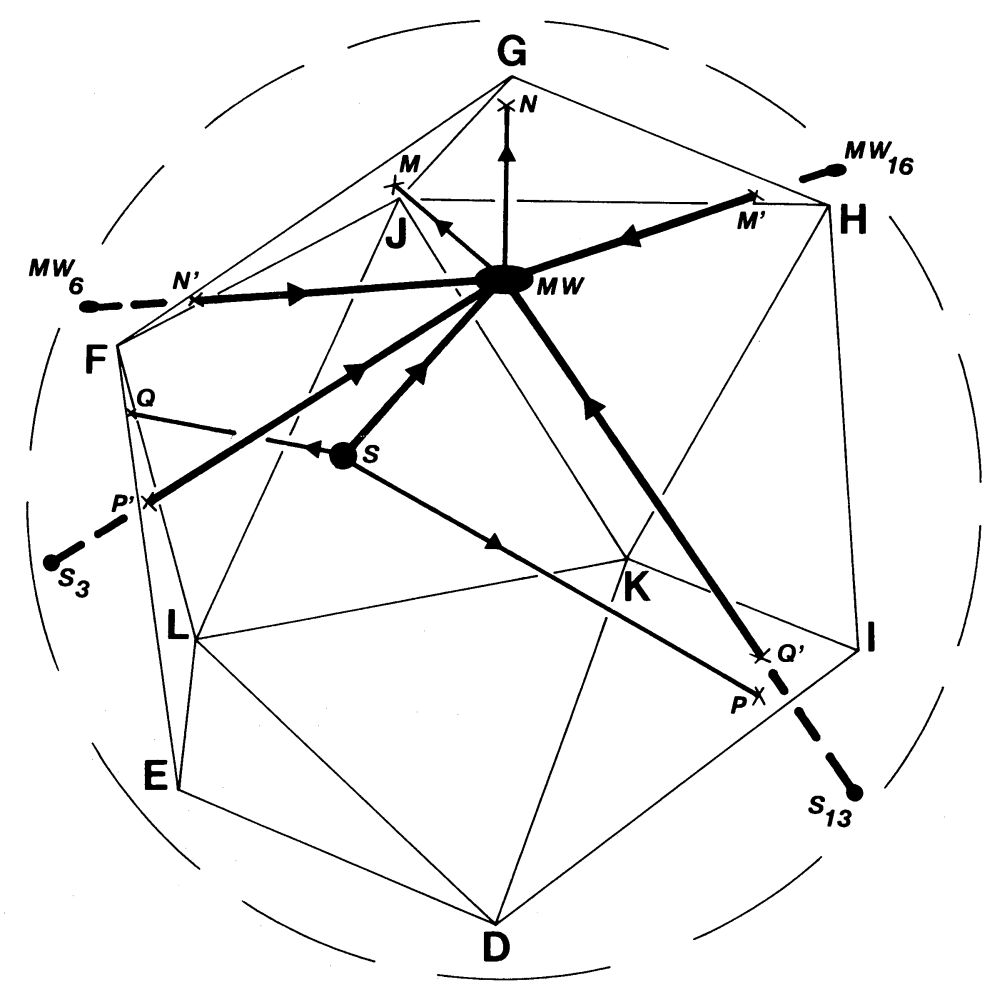

FIG. 2.-The formation of multiple images is illustrated with two sources; our own Galaxy (MW) has two images $\mathrm{MW}_{6}$ and $\mathrm{MW}_{16}$ of its old self, and source $\mathrm{S}$ has images $S, S_{3}$, and $S_{13}$. Here the position of $M W$ and the several rays do not correspond to those in the text; they were chosen for illustration and drawing convenience. The front faces of Fig. 1 are omitted here and in the next two figures. 
lat $=199.8$, lon $=-6.2$. There are three other potential images of MW with $Z<4.5, \mathrm{MW}_{5}$ at $(3.73,300.0,23.6), \mathrm{MW}_{6}$ at $(2.23,271: 8,-6.2)$, and $M W_{15}$ at $(3.73,164.6,-29.4)$.

In Figure 2 another source $S$ produces a direct image by ray S-MW. Its image $S_{k}$ in a neighbor cell is given by the action of generator $k$, and a procedure similar to the above one is used to find its parameters $(Z$, lon, lat). The details of the procedure should become clear from the discussions of Appendix B.

In general, the positions of $S$ and $S_{k}$ will not be in a noteworthy correlation like conjunction or opposition. In next paragraph the search for these specially correlated images is explained.

In this paper I am only looking for repeated images in the first layer of cells around the FP. An estimate shows that images in the second layer have $Z>4$. They are not considered here because the effort is not worthwhile in the present situation, where the model is not being fitted to data.

\section{THE SEARCH FOR IMAGES IN CONJUNCTION AND BY-PRODUCTS OF THAT SEARCH}

To determine the position of a source whose direct image is in conjunction with a second image, I found a more efficient method than that used in QGAI, $\& \mathrm{~V}$, where favorable cases were looked for along a number of fixed, tentative directions.

Referring to Figure 3, where again the position of $\mathrm{MW}$ and the drawn rays are only illustrative, let us search for conjunctions in the directions from $\mathbf{M W}$ to points on each face $\mathbf{N}$. In this context the face $\mathbf{N}$ that determines this set of directions is called Search Region N (SR N). For example, Search Region 16 refers to directions from $\mathrm{MW}$ to points on face GJH. The idea is to set a mesh of lines on GJH, as to allow a parameterization $(u, v)$ of its points, as explained in Appendix B. This is also a parameterization of directions in SR 16. To find the suitable position we choose an initial direction $(u, v)$ of SR 16; this line is moved by $G_{6}$ into a line through $\mathrm{MW}_{6}$ and $\mathrm{M}$; then we calculate the (Euclidean) distance $d(u, v)$ between these lines (see McConnell 1957, pp. 68-74, for example); by keeping $u$ fixed, a solution $v$ of the equation $d(u, v)=0$ is sought, and the point where the lines thus meet is determined as $S$, and we have a self-crossing geodesic. The details are given in Appendix B.

As seen from MW, $\mathrm{S}$ may be either in a direction toward face 6 , or in an opposite direction. In the first case we have the situation of Figure 3, where the conjunct image $S_{16}$ is produced by ray $\mathbf{S}\left(\mathbf{M}=\mathbf{M}^{\prime}\right) \mathbf{M W}$, in the second we get an opposite image, as $S_{3}$ in Figure 4. Once $S$ is determined, one may find other images correlated to it, such as $S_{3}$ and $S_{6}$ in Figure 3. Then we remake the search for a different $u$, and it is possible, by refining the values of parameter $u$, or sometimes by redefining the parameter mesh, to find a range of solutions for each search region.

The 20 Search Regions were thus explored for conjunctions and oppositions, but images with $Z>4.5$ were discarded. Only Search Regions 5, 6, 15, 16 gave interesting results, which are plotted in Figure 5. A number of representative positions for both sources and correlated images is given in Table 2, together with the corresponding redshifts. (Other positions, with higher precision than obtainable from Fig. 5, as well as their redshifts, can be obtained by interpolation on the numbers of Table 2.)

Let it be clear that the segments of Figure 5 are loci of potential positions of sources or images. The topological structure only guarantees the existence of multiple geodesic paths. The real existence of an adequate source at these points is an astrophysical matter: statistically there is a chance that some

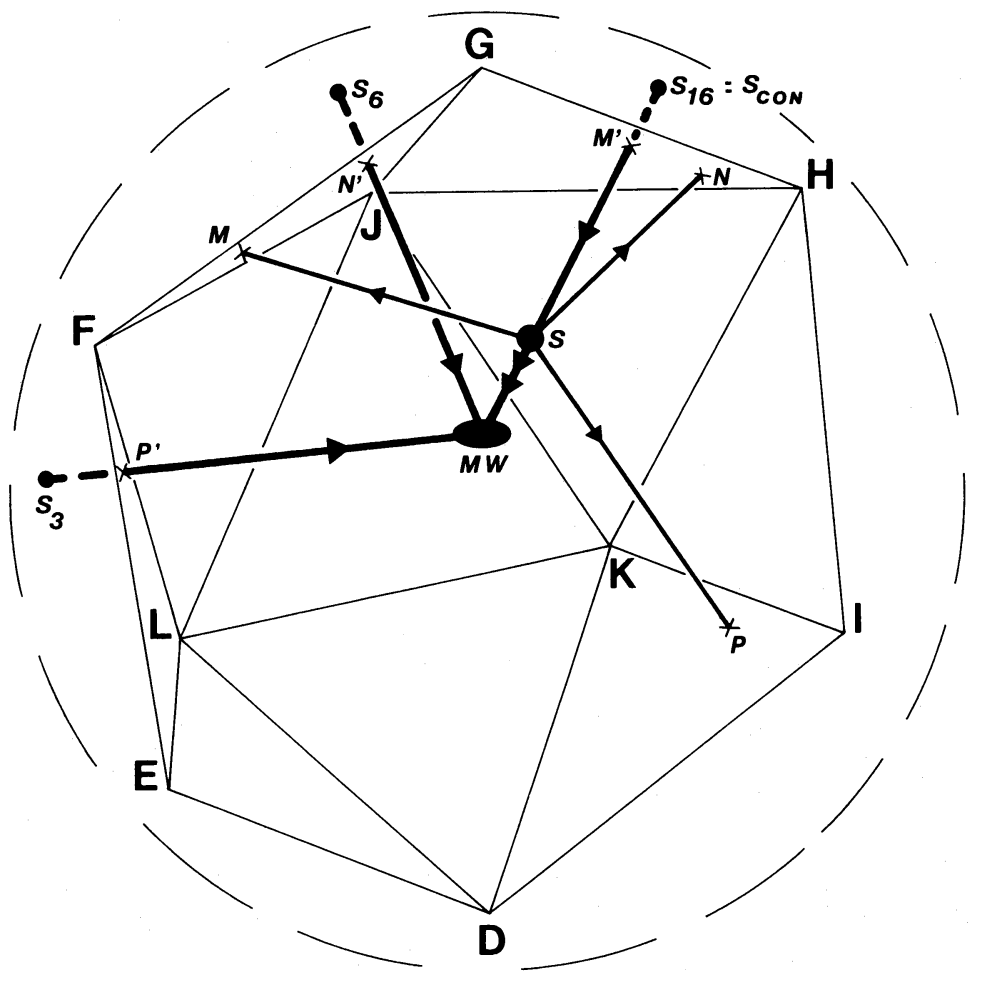

FIG. 3. - Four images of a source, $S, S_{3}, S_{6}, S_{16}$. Their production is similar to those of Fig. 2, but $S$ here has been determined so that it has an image $S_{16}$ in conjunction with the direct image. As in Fig. 2, the points and rays here are illustrative, not actual examples from the text. 


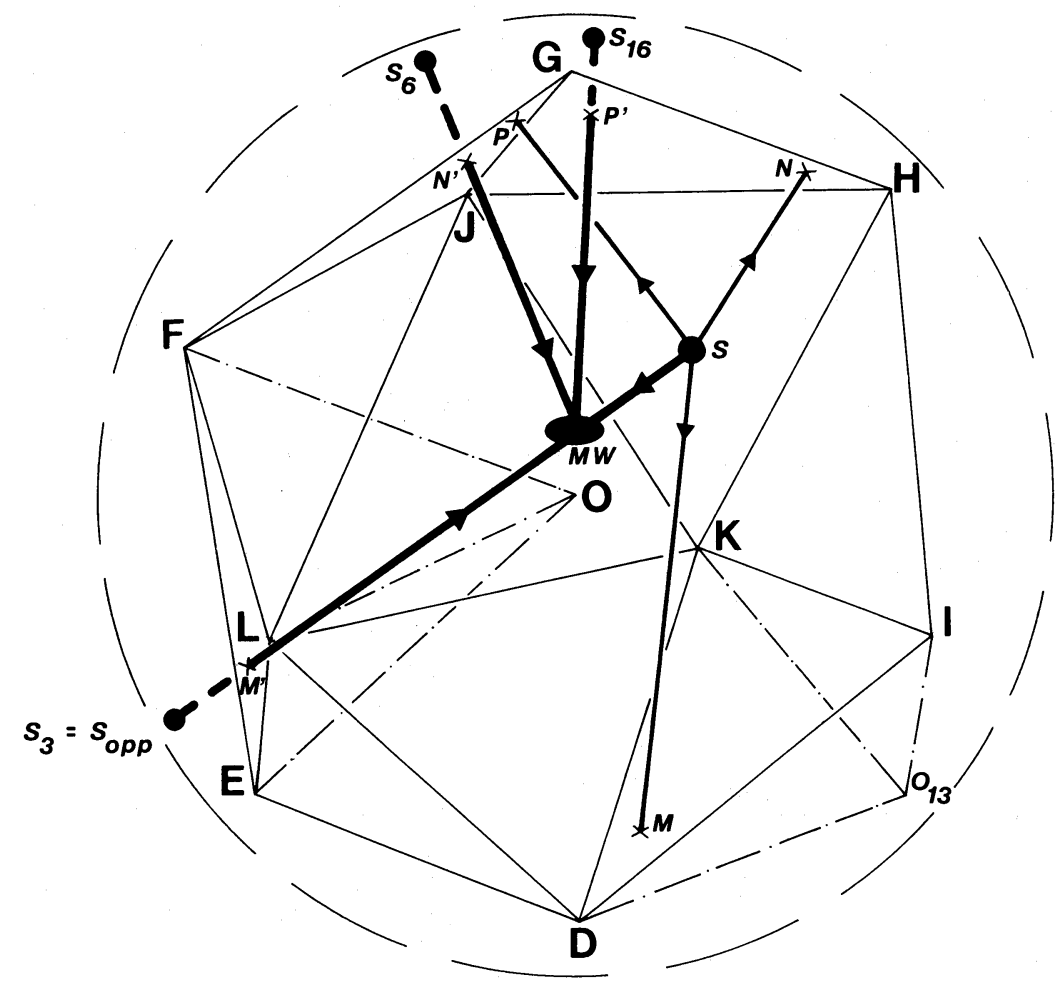

Fig. 4.-As in Fig. 3, four images of a source are illustrated, but now $S$ was found opposite to the search direction. So we get an image $S_{3}$ opposite to $S$. Also shown are the pyramids OEFL, $\mathrm{O}_{13}$ DIK, which are used in Appendix A for the determination of generator 13 .

sources will be located along these ranges, or near them, and according to each source's history it may or may not have been radiating at the appropriate times to produce the topologically allowed images.

The by-products of this search, the other potential images besides the conjunct one, belong to the total picture: they represent "snapshots" of the source at different epochs. Consider, for example, the first row of Table 2. It might happen that we saw all listed images except the conjunct one. If nothing were blocking the latter's detection, we might infer that this source is younger than $Z=4.263$. This information, plus the recognized images of the same object at times $Z=3.136,2.342,1.938$, and 0.124 would delight evolution theorists and observers alike.

Some facts may deviate an image from a straight apparent path, like gravitational lensing and random velocities at the time of emission, so that a theoretically calculated conjunction may turn out to be really a near conjunction (see Demianski and Lapucha 1987 for an estimate of possible deviations in their similar problem). An exact conjunction could leave the older image hidden behind its source. This might be happening with presumed high- $Z$ middle images in Arp and Hazard's (1980) triplets, which are being studied by the author (see QGAI). As to local motions, this important phenomenon might happen: suppose a nearby couple of quasars (young galaxies) sent us their light at high- $Z$ time, by long paths on self-crossing geodesics; while these separate rays were moving toward Earth their sources moved toward each other to collide eventually and form a single galaxy, which would now be seen as a low- $Z$ source surrounded by its ancestors. The author has in mind the peculiar galaxy M82 and the Hoag quasars around it (Burbidge et al. 1980). Finally note that gravitational focusing should play the same role in physical conjunctions as it does in coincidental ones (see Weedman 1988, p. 91).

\section{A COMPARISON WITH OBSERVATIONAL DATA}

Before attempting a comparison with data, let me recall an aspect of this work that may not have been sufficiently emphasized. The closed hyperbolic manifold that is the spatial component of the model is locally homogeneous. This implies that neighborhoods of distinct observers are physically similar (see comment after eq. [2.8]). However, differently from Euclidean and spherical manifolds, no CHM is globally homogeneous (see Ellis 1971). So for each point (observer) of the FP there is a peculiar set of geodesic loops, although some of these sets may be similar because of partial symmetries. This was discussed in QGAI, to the effect that the postulate of homogeneity is reinterpreted as a homogeneity of images, in a large-scale sense; the distribution of cosmic images remains basically uniform, but correlations between them will in general be different for different observers (see Fagundes 1986 for an illustration of this question). My choice of Earth's position here was made in the trial and error spirit of computer simulations: many other choices were discarded since they did not come closer to produce the desired effects. How, then, is the homogeneity of images to be reconciled with the inhomogeneities of Figure 5? Actually the multiple images here derived should be blended with the bulk of QSO images to give the expected, approximately homogeneous distribution. Suppose a strictly homogeneous population of sources, for example the fundamental polyhedron divided into equal cells of $1 \mathrm{Mpc}^{3}$ volume, with exactly one source in the center of each cell is taken and evolution effects are disregarded. Then this model would predict a strictly homogeneous distribution of images (this was succinctly proved in Fagundes 1983), and eventual physical conjunctions would be only coincidental because the positions of the sources would in general not be the appropriate ones here 


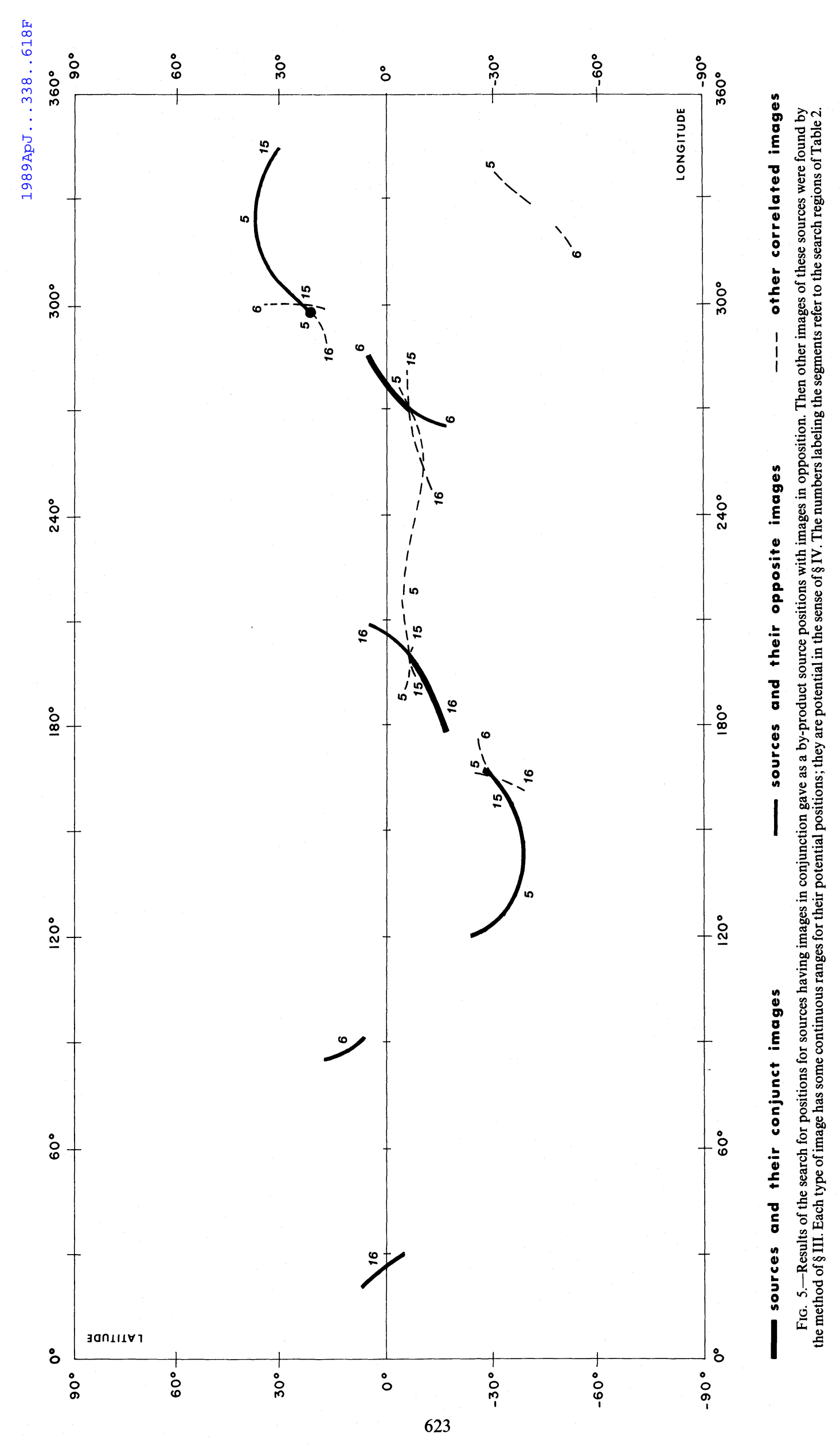

(C) American Astronomical Society - Provided by the NASA Astrophysics Data System 
TABLE 2

Potential Positions for MUltiple IMAges Of SOURCes ${ }^{a}$

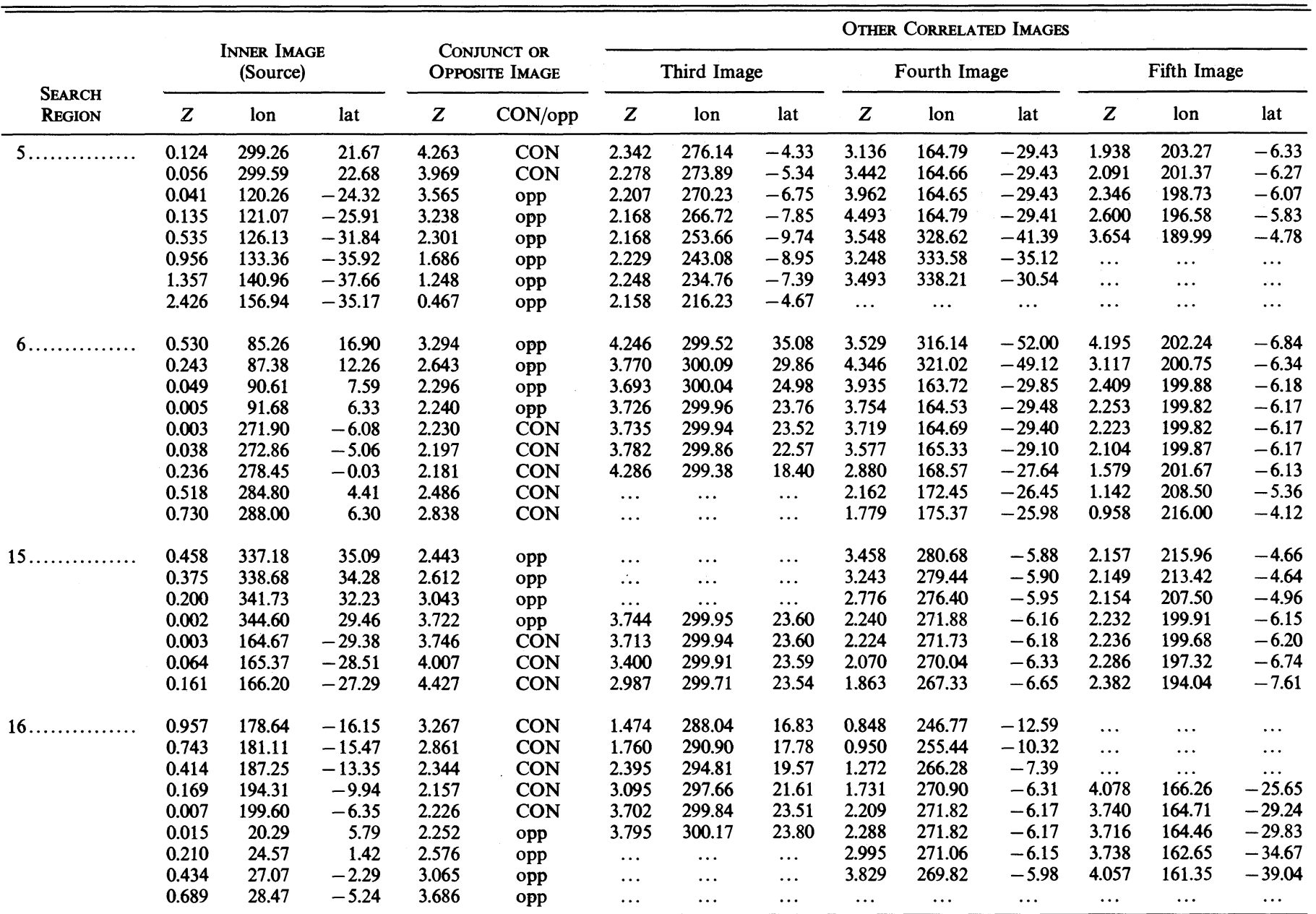

${ }^{a}$ For each search region, the positions in each column are representative of the continuous ranges of potential positions plotted in Fig. 5 . There are some

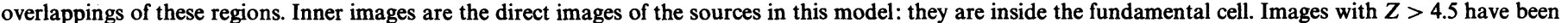
discarded.

calculated. But, of course, real sources are randomly distributed, and do evolve, and some will be found at the suitable places for physical conjunctions. So the distribution of conjuncts and other correlates will not be random, their positions being determined by the (globally inhomogeneous) properties of the CHM.

In this prototype model the favorable positions for conjunction are inside a narrow band crossing the theoretical equator. This results from the positions of the favorable pairs of faces $(5,15)$ and $(6,16)$, with respect to the observer's position MW. To show that this need not be the case in general, Table 3 lists a distribution of conjunct pairs with $\mathrm{MW}$ at the center of the icosahedron, and without the restriction $Z<4.5$. This is unrealistic, but it shows that positions for conjunct images will not necessarily be predicted to lie in a narrow band on the sky. Given the infinite number of CHMs, it may be hoped that a sufficiently small CHM will be found to meet the requirements of a fit to the data (see below).

I cannot pretend to explain here all observations relating to the quasar redshift controversy. But it is remarkable that some of their features are qualitatively, even semiquantitatively, reproduced by the results of this tentative, prototype model.

First, since the redshifts for most sources in Table 2 are in
TABLE 3

Some Potential Positions of Conjunct Images If Our Galaxy IS PLACED in the CENTER OF THE FundaMeNTAL Region

\begin{tabular}{|c|c|c|c|}
\hline \multicolumn{3}{|c|}{$\begin{array}{l}\text { INNER IMAGE } \\
\text { (Source) }\end{array}$} & \multirow{2}{*}{$\begin{array}{c}\begin{array}{c}\text { CONJUNCT } \\
\text { IMAGE }\end{array} \\
Z\end{array}$} \\
\hline$Z$ & lon & lat & \\
\hline 0.69 & 30.45 & 45.35 & 9.22 \\
\hline 0.39 & 72.68 & -16.55 & 4.81 \\
\hline 0.34 & 75.30 & -48.07 & 7.69 \\
\hline 0.36 & 109.53 & 15.63 & 7.88 \\
\hline 0.39 & 110.99 & 47.99 & 8.36 \\
\hline 0.39 & 146.99 & -6.77 & 8.36 \\
\hline 0.34 & 152.27 & -52.67 & 7.69 \\
\hline 0.69 & 175.90 & 17.91 & 9.22 \\
\hline 0.39 & 206.84 & -54.58 & 4.81 \\
\hline 1.46 & 283.62 & 53.15 & 5.25 \\
\hline 0.34 & 315.73 & 52.67 & 7.69 \\
\hline 0.39 & 321.01 & 6.77 & 8.36 \\
\hline 0.39 & 357.01 & -47.99 & 8.36 \\
\hline 0.36 & 358.47 & -15.63 & 7.88 \\
\hline
\end{tabular}

This table shows that the conjunct pairs need not be located in a narrow band, as in Fig. 5. 
the small to medium range, these sources may be either galaxies or quasars. So the probability of an actual source to be found in these places is high. But then, if that source is old enough, so is the probability that their correlated-conjunct, opposite, or other-images be also found in their respective positions, since these are a consequence of the topologicalgeometrical configuration of the model's space. This argument, coupled with suitable statistics, might explain the larger than chance occurrence of conjunctions with discordant redshifts, attributed by Arp's school to local, material links between different sources. However, the redshifts for the conjunction entries in Table 2 are overall larger by factors 2 to 3 with respect to those in Table 1 of Burbidge (1981). This is why no data fitting was attempted in this prototype. What happens is that the currently available CHMs are too big, hence the looping rays are too long, and the images are too far away. Even so, there is a tenfold improvement in this respect as we compare with the results of the QGAI study. See Gott (1980) for the likelihood that geometers will present us with CHMs sufficiently small for our purposes. The small number of regions favorable for conjunction is related to the relative simplicity of our FP. Other, more complex CHMs are being studied by geometers, but the matter is not trivial (see Thurston 1982, especially his Fig. 6, drawn from the research of $\mathbf{R}$. Riley).

Second, as a result of the proximity of the our Galaxy (MW) from the pairs of identified faces (GFB, HJK) and (FGJ, GJH) in Figure 1, the conjunctions appear in the general directions of these faces (Search Regions 5, 6, 15, 16 in Table 2), and combined with other correlated images produce the interesting line segments of potential images in Figure 5: these are irregularly distributed on the sky, as expected from the discussion above; many cross each other; and looking at Table 2 for the redshifts, one sees the possibility of a number of group patterns. This is in line with an inhomogeneous distribution of quasar images and the formation of groups (Arp 1983, $1984 a, b)$. For example, several lines converge near (lon, lat) = $\left(272^{\circ},-6^{\circ}\right)$. From Table 2 , we see that this point is the potential position of three images with $Z \approx 2.2$, whose sources have small redshifts and are located approximately at $\left(200^{\circ},-6^{\circ}\right)$, $\left(272^{\circ},-6^{\circ}\right)$, and $\left(344^{\circ},-29^{\circ}\right)$. This unexpected result reminds the author of Arp's report that QSOs with $Z \sim 2$ are concentrated in an area of the sky around the center of the Local Group of galaxies.

The question of the periodicity in the distribution of quasar redshifts (see Burbidge 1981) has not been addressed in this paper. Note that I have not looked for images beyond the first layer of cells around the fundamental region. If we can find a CHM with small enough dimensions, then a search for repeated images including the second and third layers might produce the mentioned periodicity and also give a topological interpretation for the rich groups of QSOs around bright galaxies, such as those compiled by Burbidge (1981) and by Arp (1983). Hopefully, it might even provide the kind of small spaces needed by Gott's (1980) and Ellis and Schreiber's (1986) alternative explanations for the homogeneity of the matter distribution in the universe.

Let me add that the idea of the closure of space has a larger theoretical relevance than the prediction of multiple images emphasized in the present application (see Wheeler 1964; Goncharov and Bytsenko 1988, for example). So our model has a wider interest than the stated aim of solving the quasar redshift controversy.

This work was partly supported by Conselho Nacional de Desenvolvimento Científico e Tecnológico and Financiadora de Estudos e Projetos, agencies of the Brazilian Government.

I am particularly grateful to my sister, Dr. Wilma F. FigàTalamanca, for her substantial financial help.

\section{APPENDIX A}

\section{THE FUNDAMENTAL POLYHEDRON AND THE GENERATORS OF THE TESSELLATION}

Our FP is a regular icosahedron in $\mathbf{H}^{3}$. Its dimensions are fixed by the constraint that it must tessellate the space in order that it be transformed into a closed hyperbolic space upon the gluing of faces (Poincarés theorem). This implies that the dihedral angles are $120^{\circ}$. Then (see Coxeter 1968, pp. 199-214) the radius of the circumscribing sphere is $\chi=\cosh ^{-1}\left(\tau^{3} / 2\right), \tau=(\sqrt{5}+1) / 2$, or $\chi \approx 1.3826$; the radius of the inscribed sphere is $\psi=\cosh ^{-1}[\sqrt{3} \tau / 2] \approx 0.8683$, and the edges have length $2 \psi$. (Although $I$ often refer to numbers by approximations of a few digits, the calculations were performed to the full precision of the used computer: 15 digits internally, 12 for input-output.)

From these results, plus an information on the regular Euclidean icosahedron (Euclid 1956, p. 492), I obtained the coordinates, in Klein's model, of the vertices of the FP in the position of Figure 1: the radial coordinate is tanh $\chi \approx 0.8815$, and the angular coordinates are given in Table 4 . We get coordinates $x_{i}$ by equations $(2.6 \mathrm{a})-(2.6 \mathrm{c})$.

A rigid motion in $\mathbf{H}^{3}$, such as those that move the FP onto replicas of itself, is elegantly performed through a one-to-one correspondence between these actions and the (proper, orthochronous) Lorentz transformations of Minkowski space (see Efimov $1980, \S 202$; Fagundes 1986 , and references therein). For this purpose we represent a point in $\mathbf{H}^{3}$ by $\mathrm{Minkowski}$ coordinates $X_{m}$, $m=1-4$, subject to the condition $X_{1}^{2}+X_{2}^{2}+X_{3}^{2}-X_{4}^{2}=-1$ (compare Landau and Lifshitz 1975, $\S$ III). The relation between these coordinates and Klein's is simply $X_{i}=x_{i} X_{4}, i=1-3, X_{4}=\cosh \chi$.

Consider the determination of $G_{13}$, which moves the FP into a neighbor replica of itself. We know from $\S$ III that $G_{13}($ face $3)=$ face 13, and from Figure 4 we see that $G_{13} O=O_{13}$, where $O_{13}$ is the mirror image of $O$ on face 13 , and so its coordinates can be calculated. The Minkowskian coordinates of $O$ are $(0,0,0,1)$, and those of vertices E, F, L, D, I, K can be calculated from Table 3 . So we get a matrix equation for $G_{13}$ :

$$
G_{13} M_{3}=M_{13}
$$


TABLE 4

ANGUlar CoOrdinates of THE Vertices of the Fundamental POLYHEDRON

\begin{tabular}{|c|c|c|}
\hline Vertex & $\theta$ & $\varphi$ \\
\hline G & $0^{\circ}$ & \\
\hline ...... & $\mathbf{W}^{\mathbf{a}}$ & $0^{\circ}$ \\
\hline $\mathrm{C}$ & W & $72^{\circ}$ \\
\hline $\mathbf{H}$ & W & $144^{\circ}$ \\
\hline ............. & W & $216^{\circ}$ \\
\hline (n............... & W & $288^{\circ}$ \\
\hline ............ & $180^{\circ}-\mathrm{W}$ & $36^{\circ}$ \\
\hline ….................... & $180^{\circ}-W$ & $108^{\circ}$ \\
\hline (n) & $180^{\circ}-W$ & $180^{\circ}$ \\
\hline 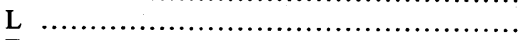 & $180^{\circ}-\mathrm{W}$ & $252^{\circ}$ \\
\hline E & $180^{\circ}-W$ & $324^{\circ}$ \\
\hline D & $180^{\circ}$ & $\ldots$ \\
\hline
\end{tabular}

angle $W=\tan ^{-1} 2 \approx 63.4349$.

where $M_{3}$ results from writing the Minkowski coordinates of O, E, F, L as columns; to four decimals,

$$
M_{3}=\left(\begin{array}{rrrr}
0 & 0.8938 & 0.3414 & -0.3414 \\
0 & -0.6494 & -1.0508 & -1.0508 \\
0 & -0.5524 & 0.5524 & -0.5524 \\
1 & 1.4013 & 1.4013 & 1.4013
\end{array}\right) \text {, }
$$

and similarly, with points $\mathrm{O}_{13}, \mathrm{D}, \mathrm{I}, \mathrm{K}$,

$$
M_{13}=\left(\begin{array}{rcrc}
-1.3510 & 0 & -0.3414 & -1.1048 \\
0.9816 & 0 & 1.0508 & 0 \\
-2.1860 & -1.2352 & -0.5524 & -0.5524 \\
2.9271 & 1.4013 & 1.4013 & 1.4013
\end{array}\right) \text {. }
$$

Finally, $G_{13}=M_{13} M_{3}^{-1}$ gives $G_{13}$ as listed below. Generator 3 can be calculated by the same method or by matrix inversion, $G_{3}=G_{13}^{-1}$ (or both, for checking). The same is true of the other pairs $G_{k}, G_{k+10}, k=1-10$.

In the text we also need Kleinian coordinates centered on Earth, but with axes parallel to the O-centered ones. Let $\beta=\tanh 0.75$, $\gamma=\cosh 0.75$. In Minkowski space the transformation is equivalent to an active motion that takes point $(0,0, \beta \gamma, \gamma)$ to $(0,0,0,1)$, takes the latter to $(0,0,-\beta \gamma, \gamma)$, and preserves the coordinates $X_{1}, X_{2}$ of any moved point. The result is the familiar Lorentz transformation $X_{1}^{\prime}=X_{1}, X_{2}^{\prime}=X_{2}, X_{3}^{\prime}=\gamma\left(X_{3}-\beta X_{4}\right), X_{4}^{\prime}=\gamma\left(X_{4}-\beta X_{3}\right)$, and the new Klein coordinates are $x_{i}^{\prime}=X_{i}^{\prime} / X_{4}^{\prime}$.

The 20 generator matrices follow (to six decimals). They are identified by their action on the faces of the FP: generator $\mathrm{N}$ takes onto face $\mathrm{N}$ the face to be glued to it.

Generator 1: BGC $\rightarrow$ ADI

Generator 11: ADI $\rightarrow$ BGC

$\left(\begin{array}{rrrr}0.447214 & -0.850651 & -0.585410 & 0.516054 \\ -0.525731 & 0.000000 & -1.801707 & 1.588251 \\ 1.532624 & 1.113516 & 1.479837 & -2.186041 \\ -1.351047 & -0.981593 & -2.186041 & 2.927051\end{array}\right)$

$\left(\begin{array}{rrrr}0.447214 & -0.525731 & 1.532624 & 1.351047 \\ -0.850651 & 0.000000 & 1.113516 & 0.981593 \\ -0.585410 & -1.801707 & 1.479837 & 2.186041 \\ -0.516054 & -1.588251 & 2.186041 & 2.927051\end{array}\right)$

Generator 2: $\mathrm{ABC} \rightarrow \mathrm{ICA}$

$\left(\begin{array}{rrr}-1.085410 & -0.262866 & 0.670820 \\ -1.964167 & -1.809017 & -0.688191 \\ 0.861803 & -0.425325 & 0.585410 \\ -2.186041 & -1.588251 & -0.516054\end{array}\right.$

0.834993
2.569845
-0.516054
2.927051

Generator 12: ICA $\rightarrow$ ABC

Generator 3: DIK $\rightarrow$ EFL

$\left(\begin{array}{rrrr}-1.085410 & -1.964167 & 0.861803 & 2.186041 \\ -0.262866 & -1.809017 & -0.425325 & 1.588251 \\ 0.670820 & -0.688191 & 0.585410 & 0.516054 \\ -0.834993 & -2.569845 & 0.516054 & 2.927051\end{array}\right)$

$\left(\begin{array}{rrrr}1.256231 & -0.262866 & 0.223607 & 0.834993 \\ -1.113516 & 0.809017 & -2.389493 & -2.569845 \\ -0.085410 & 1.113516 & -0.138197 & -0.516054 \\ 1.351047 & -0.981593 & 2.186041 & 2.927051\end{array}\right) \quad\left(\begin{array}{rrrr}1.256231 & -1.113516 & -0.085410 & -1.351047 \\ -0.262866 & 0.809017 & 1.113516 & 0.981593 \\ 0.223607 & -2.389493 & -0.138197 & -2.186041 \\ -0.834993 & 2.569845 & 0.516054 & 2.927051\end{array}\right)$ 
Generator 4: KLD $\rightarrow$ DEL

$\left(\begin{array}{rrrr}0.223607 & -0.688191 & 0.861803 & 0.516054 \\ -1.538842 & -0.500000 & -0.951057 & -1.588251 \\ -0.638197 & -1.113516 & -2.032624 & -2.186041 \\ 1.351047 & 0.981593 & 2.186041 & 2.927051\end{array}\right)$

Generator 5: HJK $\rightarrow$ GFB

$\left(\begin{array}{rrr}1.256231 & -0.262866 & -1.085410 \\ -1.113516 & 0.809017 & -0.262866 \\ 2.341641 & 0.525731 & -0.138197 \\ 2.702095 & 0 & -0.516054\end{array}\right.$

Generator 6: GJH $\rightarrow$ FGJ

$\left(\begin{array}{rrr}0.223607 & -0.688191 & 0.861803 \\ -1.538842 & -0.500000 & 0.951057 \\ 1.170820 & -0.525731 & -2.032624 \\ 1.669986 & 0.000000 & -2.186041\end{array}\right.$

Generator 7: LJF $\rightarrow \mathrm{ABE}$

$\left(\begin{array}{rcr}0.723607 & 2.752764 & -0.447214 \\ -0.525731 & 0 & -0.850651 \\ -0.947214 & -0.162460 & 0.585410 \\ 0.834993 & 2.569845 & -0.516054\end{array}\right.$

Generator 8: CGH $\rightarrow$ FEB

$\left(\begin{array}{rrrr}-0.085410 & -1.639247 & -1.756231 & 2.186041 \\ -1.113516 & 0.809017 & 1.275976 & -1.588251 \\ -0.138197 & 0.425325 & -1.032624 & 0.516054 \\ 0.516054 & -1.588251 & -2.186041 & 2.927051\end{array}\right)$

Generator 9: KIH $\rightarrow$ AED

$\left(\begin{array}{rrcc}1.532624 & -1.113516 & -0.447214 & 1.669986 \\ -0.587785 & -0.809017 & 0 & 0 \\ -1.756231 & 1.275976 & -1.032624 & -2.186041 \\ 2.186041 & -1.588251 & 0.516054 & 2.927051\end{array}\right)$

Generator 10: CHI $\rightarrow$ JKL

$\left(\begin{array}{rrrr}-0.585410 & 2.327438 & -0.138197 & -2.186041 \\ -0.951057 & 1.309017 & 0.951057 & -1.588251 \\ 0.670820 & 0.688191 & 0.585410 & -0.516054 \\ 0.834993 & -2.569845 & -0.516054 & 2.927051\end{array}\right)$
Generator 14: DEL $\rightarrow$ KLD

$\left(\begin{array}{rrrr}0.223607 & -1.538842 & -0.638197 & -1.351047 \\ -0.688191 & -0.500000 & -1.113516 & -0.981593 \\ 0.861803 & -0.951057 & -2.032624 & -2.186041 \\ -0.516054 & 1.588251 & 2.186041 & 2.927051\end{array}\right)$

Generator 15: GFB $\rightarrow$ HJK

$\left(\begin{array}{rrrr}1.256231 & -1.113516 & 2.341641 & -2.702095 \\ -0.262866 & 0.809017 & 0.525731 & -0.000000 \\ -1.085410 & -0.262866 & -0.138197 & 0.516054 \\ -1.351047 & 0.981593 & -2.186041 & 2.927051\end{array}\right)$

Generator 16: FGJ $\rightarrow$ GJH

$\left(\begin{array}{rrrc}0.223607 & -1.538842 & 1.170820 & -1.669986 \\ -0.688191 & -0.500000 & -0.525731 & 0 \\ 0.861803 & 0.951057 & -2.032624 & 2.186041 \\ 0.516054 & 1.588251 & -2.186041 & 2.927051\end{array}\right)$

Generator 17: ABE $\rightarrow$ LJF

$\left(\begin{array}{rrrr}0.723607 & -0.525731 & -0.947214 & -0.834993 \\ 2.752764 & 0.000000 & -0.162460 & -2.569845 \\ -0.447214 & -0.850651 & 0.585410 & 0.516054 \\ -2.702095 & -0.000000 & 0.516054 & 2.927051\end{array}\right)$

Generator 18: FEB $\rightarrow \mathrm{CGH}$

$\left(\begin{array}{rrrr}-0.085410 & -1.113516 & -0.138197 & -0.516054 \\ -1.639247 & 0.809017 & 0.425325 & 1.588251 \\ -1.756231 & 1.275976 & -1.032624 & 2.186041 \\ -2.186041 & 1.588251 & -0.516054 & 2.927051\end{array}\right)$

Generator 19: AED $\rightarrow \mathrm{KIH}$

$\left(\begin{array}{rrrr}1.532624 & -0.587785 & -1.756231 & -2.186041 \\ -1.113516 & -0.809017 & 1.275976 & 1.588251 \\ -0.447214 & 0.000000 & -1.032624 & -0.516054 \\ -1.669986 & -0.000000 & 2.186041 & 2.927051\end{array}\right)$

Generator 20: JKL $\rightarrow$ CHI

$\left(\begin{array}{rrrr}-0.585410 & -0.951057 & 0.670820 & -0.834993 \\ 2.327438 & 1.309017 & 0.688191 & 2.569845 \\ -0.138197 & 0.951057 & 0.585410 & 0.516054 \\ 2.186041 & 1.588251 & 0.516054 & 2.927051\end{array}\right)$

\section{APPENDIX B}

\section{DETERMINATION OF SELF-CROSSING SPACE GEODESICS}

The search for self-crossing geodesics of space was implemented in a small but efficient computer, Hewlett-Packard's HP 71B, enhanced by a Math ROM module that includes hyperbolic functions and their inverse, block matrix operations, and an equationsolving function. The results were checked by an independent program, as explained below. The several programs and subprograms approximately correspond to the algebraic formalism of this Appendix.

Consider two identified faces in the FP, such as GFB (face 5) and HJK (face 15). Figure 6 shows these faces as well as Earth's position E (= MW in Figs. 1-4). We will now see how to look for the conjunct or opposite images in Search Region 15, defined (see $\S$ IV) as the set of directions through $E$ and a point on HJK. Such a point, say $P$ in Figure 6, is parameterized by projecting it from 


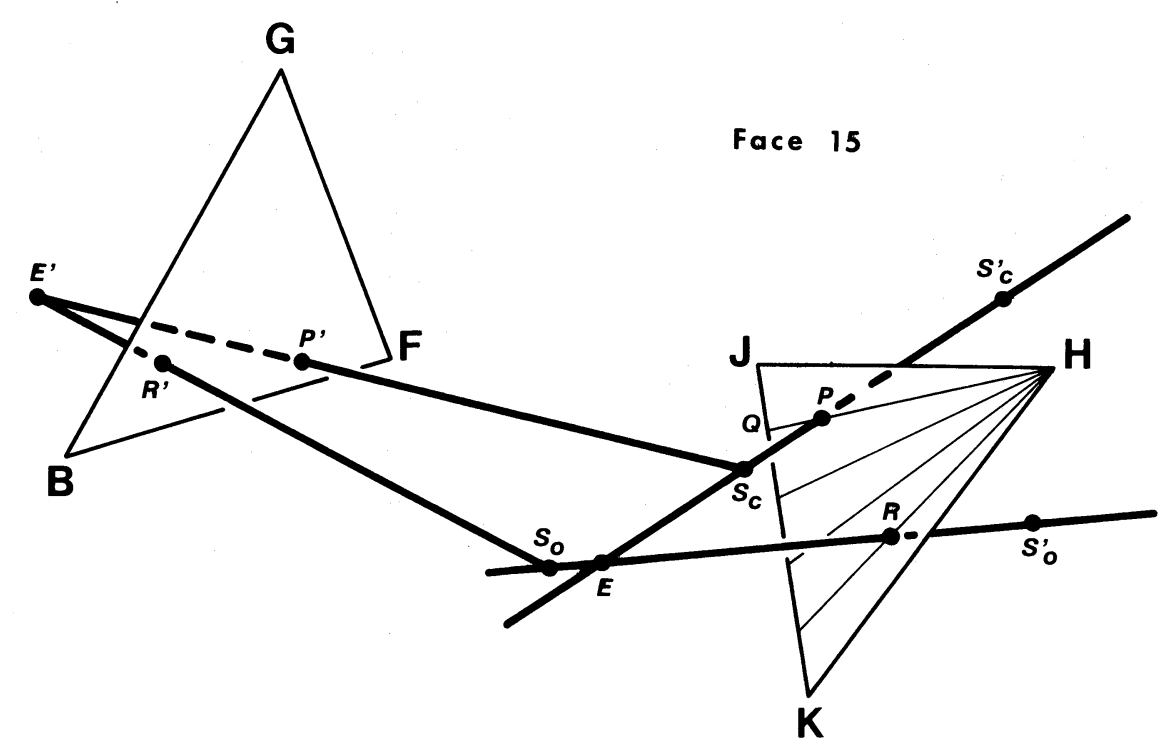

FIG. 6. -The method of finding self-crossing geodesics in the CHM. Search lines EP, ER are transformed into lines $E^{\prime} P^{\prime}, E^{\prime} R^{\prime}$, and a solution is found when the distance between original and moved lines is zero.

vertex $\mathrm{H}$ into $\mathrm{Q}$ on basis $\mathrm{JK}$, and then joining $\mathrm{Q}$ and $\mathrm{H}$. The parameters are the fractions of $\mathrm{JK}$ and $\mathrm{QH}$ determined by $\mathrm{Q}$ and $\mathrm{P}$ : $u=\mathrm{JQ} / \mathrm{JK}, v=\mathrm{QP} / \mathrm{QH}$.

Let the Minkowski coordinates of any point A be denoted by $\mathrm{A}(m), m=1-4$, the column matrix they form by $A$, and the Kleinian coordinates by $a$ or $a(i), i=1-3$. Then points $\mathrm{Q}, \mathrm{P}$ above defined have the coordinates

$$
\begin{aligned}
& q(i)=j(i)+u[k(i)-j(i)], \\
& p(i)=q(i)+v[h(i)-q(i)] .
\end{aligned}
$$

For the geometry of lines in Euclidean space I shall use McConnell's (1957, pp. 68-74) textbook. Line EP is represented by the equation

$$
x(i)=e(i)+c(i) s
$$

where

$$
c(i)=\frac{p(i)-e(i)}{|\boldsymbol{p}-\boldsymbol{e}|}
$$

Since in our CHM point $P$ is identical to a point $P^{\prime}$ on face 5 (GFB), line EP is continued inward by a segment of a line $E^{\prime} P^{\prime}$ determined as follows by the generator matrix $G_{5}: \mathrm{E}^{\prime}=G_{5} \mathrm{E}, \mathrm{P}^{\prime}=G_{5} \mathrm{P}$, where $\mathrm{E}$ is the column matrix corresponding to Earth's coordinates $(0,0, \sinh 0.75$, cosh 0.75$)$, and $P$ is obtained from $p$ as indicated in Appendix A. The Klein coordinates of the moved points are $e^{\prime}(i)=\mathrm{E}^{\prime}(i) / \mathrm{E}^{\prime}(4), p^{\prime}(i)=\mathrm{P}^{\prime}(i) / \mathbf{P}^{\prime}(4)$, and the equation for line $\mathrm{E}^{\prime} \mathbf{P}^{\prime}$ is

$$
x^{\prime}(i)=e^{\prime}(i)+c^{\prime}(i) s^{\prime},
$$

with

$$
c^{\prime}(i)=\frac{p^{\prime}(i)-e^{\prime}(i)}{\left|p^{\prime}-e^{\prime}\right|}
$$

Lines $E P, E^{\prime} \mathbf{P}^{\prime}$ have their closest approach for parameters

$$
s=\frac{\left(c^{\prime} \cos A-c\right) \cdot\left(e-e^{\prime}\right)}{\sin ^{2} A}
$$

on EP, and

$$
s^{\prime}=\frac{\left(c \cos A-c^{\prime}\right) \cdot\left(e^{\prime}-e\right)}{\sin ^{2} A}
$$


on $\mathrm{E}^{\prime} \mathrm{P}^{\prime}$, where $A=\cos ^{-1}\left(c \cdot c^{\prime}\right)$. The distance between the lines is

$$
d(u, v)=\frac{\left(\boldsymbol{e}-\boldsymbol{e}^{\prime}\right) \cdot\left(\boldsymbol{c} \times \boldsymbol{c}^{\prime}\right)}{\sin A} .
$$

I chose a number of values for parameters $u$ (in the first, gross search $u=0.1 n, n=0-10$ ) and looked for a solution $v$ of the equation $d(u, v)=0$ for each value of $u$. This was done numerically (to $10^{-12}$ precision) by the function FNROOT of the mentioned Math module. Solutions $v$ smaller than 0 or greater than 1 were discarded since they correspond to directions outside the target face (see Fig. 6 and equations [B1a]-[B1b]). The point $\boldsymbol{x}=\boldsymbol{x}^{\prime}$ of intersection was then checked for insideness: by forming pyramids with $\boldsymbol{x}$ as apex and each face in succession as basis, their oriented volumes should have appropriate signs for $x$ to be inside the FP, namely a positive sign for faces 1-10, a negative sign for faces 11-20.

If $x$ passes these tests, then we have two possibilities (besides $s=0$, which corresponds to the images of MW discussed in $\S$ III):

1. $s>0$.-Point $x$ is situated like $S_{c}$ in Figure 6. Line segment $E^{\prime} S_{c}$ is then moved by $G_{15}$. But then $E^{\prime}$ comes back to $E$, and the segment will lie on line EP, since $G_{15}$ is the opposite of motion $G_{5}$. Point $S_{c}$ goes to $S_{c}^{\prime}=G_{15} S_{c}$. Now, the hyperbolic length of loop $\mathrm{S}_{c}\left(\mathrm{P}^{\prime}=\mathrm{P}\right) \mathrm{E}$ is length $\left(\mathrm{S}_{c} \mathrm{P}^{\prime}\right)+$ length $(\mathrm{PE})$. Since $G_{5}$ is a rigid motion, length $\left(\mathrm{S}_{c} \mathrm{P}^{\prime}\right)=$ length $\left(\mathrm{S}_{c}^{\prime} \mathrm{P}\right)$, and so the length of the loop is the same as that of the apparent ray $S_{c}^{\prime} E$. This shows that $S_{c}^{\prime}$ is indeed the position of an image in conjunction with a source at $S_{c}$.

2. $s<0$. - The same reasoning applies to a source at a point like $S_{o}$ in Figure 6 , in opposition to an image at $S_{o}^{\prime}$. The ray path is $S_{0}\left(R^{\prime}=R\right) E$, of the same length as $S_{o}^{\prime} E$.

Once the coordinates $x$ of $S\left(S_{c}\right.$ or $\left.S_{o}\right)$ and $y$ of $S^{\prime}\left(S_{c}^{\prime}\right.$ or $\left.S_{o}^{\prime}\right)$ were determined, a subprogram was called to convert them into parameters $\left(Z\right.$, lon, lat). This was done by first going to E-centered coordinates $\boldsymbol{x}^{\prime}, \boldsymbol{y}^{\prime}$ (see $\S$ II and Appendix A), and then equation (2.8) gives $\chi^{\prime}\left(\boldsymbol{x}^{\prime}\right), \chi^{\prime}\left(\boldsymbol{y}^{\prime}\right)$. At this point we check for the physical meaning of $\boldsymbol{y}^{\prime}$; it should be inside the horizon sphere, that is, $\chi^{\prime}\left(y^{\prime}\right)$ should be smaller than $\eta_{0}=\cosh ^{-1}(2 / \Omega-1) \approx 3.6369$. Finally we calculate $Z(S), Z\left(S^{\prime}\right)$ by equation $(2.5)$. If $Z(S)>4.5$ or $Z\left(\mathrm{~S}^{\prime}\right)>4.5$, then the solution was discarded.

An independent program, implemented both on the HP 71B and on another microcomputer, checked the calculated results and then obtained the other correlated images of Table 2 . This program took parameters $(Z$, lon, lat) for $S$ as input, converted them to E-centered $x^{\prime}$, then to E-centered $X^{\prime}$, and then to O-centered $X$ by the inverse of the Lorents transformation defined at the end of Appendix A. From $X$ one obtains the images by the actions $X_{k}=G_{k} X, k=1-20$, and, proceeding in the opposite direction with the steps above, $X_{k} \rightarrow X_{k}^{\prime} \rightarrow x_{k}^{\prime} \rightarrow\left(Z_{k}\right.$, lon lat $\left._{k}\right)$. One of these should correspond to the formerly found $S^{\prime}$.

As already stated in $\S \mathrm{IV}$, I found a range of solutions for each of four search regions, SR 5, 6, 15, and 16, which are plotted in Figure 5. Some representative points for these ranges are given in Table 2.

\section{REFERENCES}

Arp, H. 1983, preprint. $1984 a$ Ap. J.(Letters), 277, L27. $1984 b$, J. Astr. Ap., 5, 31.

Arp, H., and Hazard, C. 1980, Ap. J., 240, 726

Best, L. A. 1971, Canadian J. Math., 23, 451.

Burbidge, G. 1981, in Proc. 10th Texas Symposium on Relativistic Astrophysics, ed. R. Ramaty and F. C. Jones (Ann. NY Acad. Sci., 375, 123).

Burbidge, E. M., Junkkarinen, V. T., Koski, A. T., Smith, H. E., and Hoag, A. A. 1980, Ap. J.(Letters), 242, L55.

Coxeter, H. S. M. 1965, Non-Euclidean Geometry (5th ed.; Toronto: University of Toronto Press).

1968, Twelve Geometric Essays (Carbondale and Edwardsville: Southern Illinois University Press).

Demianski, M., and Lapucha, M. 1987, M.N.R.A.S., 224, 527.

Efimov, N. V. 1980, Higher Geometry (Moscow: Mir).

Ellis, G. F. R. 1971, Gen. Rel. Grav., $2,7$.

Ellis, G. F. R., and Schreiber, G. 1986, Phys. Letters, A115, 97.

Euclid. 1956, Elements, Vol. 3, ed. T. L. Heath (New York: Dover).

Fagundes, H. V. 1983, Phys. Rev. Letters, 51, 517.
Fagundes, H. V. 1985, Ap. J., 291, 450.

1986, in Proc. 4th Marcel Grossman Meeting on General Relativity, ed. R. Ruffini (Amsterdam: Elsevier), p. 1559.

Fagundes, H. V., and Wichoski, W. F. 1987, Ap. J. (Letters), 322, L5.

Fang, L. Z., and Sato, H. 1983, Comm. Theoret. Phys. (China), 2, 1055.

Goncharov, Y. P., and Bytsenko, A. A. 1988, Astrophysics, 27, 422.

Gott, J. R. III. 1980, M.N.R.A.S., 193, 153.

Hut, P., and Sussman, G. J. 1987, Sci. Am., 257, No. 4, 137.

Landau, L., and Lifshitz, E. M. 1975, Classical Theory of Fields (4th ed.; Oxford: Pergamon).

McConnell, A. J. 1957, Applications of Tensor Analysis (New York: Dover).

Seifert, H and Threlfall, W. 1980, A Textbook of Topology, ed. J. S. Birman and J. Eisner (New York: Academic).

Sokolov, D. D., and Shvartsman, V. F. 1974, Soviet Phys.-JETP, 39, 196.

Thurston, W. P. 1982, Bull. Am. Math. Soc. (N.S.), 6, 357.

Weedman, D. W. 1988, Quasar Astronomy (Cambridge: Cambridge University Press).

Wheeler, J. A. 1964, in Gravitation and Relativity, ed. H.-Y. Chiu and W. F. Hoffman (New York: Benjamin), p. 303.

Helio V. Fagundes: Instituto de Física Teórica, Universidade Estadual Paulista, Rua Pamplona, 145, 01405 Sã0 Paulo, SP, Brazil 